\title{
Atomic size effects studied by transport in single silicide nanowires
}

\author{
I. Miccoli, ${ }^{1}$ F. Edler, ${ }^{1}$ H. Pfnür, ${ }^{1,2}$ S. Appelfeller, ${ }^{3}$ M. Dähne, ${ }^{3}$ K. Holtgrewe, ${ }^{4}$ \\ S. Sanna, ${ }^{4}$ W. G. Schmidt, ${ }^{4}$ and C. Tegenkamp ${ }^{1,2, *}$ \\ ${ }^{1}$ Institut für Festkörperphysik, Leibniz Universität Hannover, Appelstraße 2, 30167 Hannover, Germany \\ ${ }^{2}$ Laboratorium für Nano- und Quantenengineering (LNQE), Leibniz Universität Hannover, Schneiderberg 39, 30167 Hannover, Germany \\ ${ }^{3}$ Institut für Festkörperphysik, Technische Universität Berlin, Hardenbergstraße 36, 10623 Berlin, Germany \\ ${ }^{4}$ Lehrstuhl für Theoretische Physik, Universität Paderborn, 33098 Paderborn, Germany \\ (Received 16 December 2015; revised manuscript received 11 February 2016; published 8 March 2016)
}

\begin{abstract}
Ultrathin metallic silicide nanowires with extremely high aspect ratios can be easily grown, e.g., by deposition of rare earth elements on semiconducting surfaces. These wires play a pivotal role in fundamental research and open intriguing perspectives for CMOS applications. However, the electronic properties of these one-dimensional systems are extremely sensitive to atomic-sized defects, which easily alter the transport characteristics. In this study, we characterized comprehensively $\mathrm{TbSi}_{2}$ wires grown on $\mathrm{Si}(100)$ and correlated details of the atomic structure with their electrical resistivities. Scanning tunneling microscopy (STM) as well as all transport experiments were performed in situ using a four-tip STM system. The measurements are complemented by local spectroscopy and density functional theory revealing that the silicide wires are electronically decoupled from the Si template. On the basis of a quasiclassical transport model, the size effect found for the resistivity is quantitatively explained in terms of bulk and surface transport channels considering details of atomic-scale roughness. Regarding future applications the full wealth of these robust nanostructures will emerge only if wires with truly atomically sharp interfaces can be reliably grown.
\end{abstract}

DOI: 10.1103/PhysRevB.93.125412

\section{INTRODUCTION}

The large and still growing interest in silicides, i.e., binary compounds of $\mathrm{Si}$ with electropositive elements like transition metals (TM) or rare earth (RE) elements, is driven by their unique properties for applications as well as for addressing fundamental questions [1-6]. For instance, novel ultralow resistivity (i.e., $\rho \ll 10 \mu \Omega \mathrm{cm}$ ) TM-based silicides are intended to be used as interconnects or gate electrodes for the next generation CMOS technology [7-11]. Compared to noble metals this material class provides appropriate Schottky barrier heights, thermal stability, and high robustness against electromigration processes [12]. Nonetheless, the gradual scaling of Si technology towards the sub-10-nm node demands silicide structuring beyond the capabilities of current lithographic techniques. Moreover, such nanoscale structures may be characterized by electronic properties deviating from those of the bulk silicides, requiring detailed investigations on the atomic scale.

In this respect, RE metal silicides with a hexagonal $\mathrm{AlB}_{2}$ bulk structure are of particular interest, because they offer tunable Schottky contact resistances [13] and they can grow as long but extremely thin nanowires on (100)-oriented or vicinal (111)-oriented Si substrates [14-17]. These concepts of strainor step-mediated growth are highly tempting since lithographic problems and limitations are entirely circumvented and, moreover, the metallic wires serve as important model systems to understand the effects of electronic correlation and instabilities characteristic for one-dimensional (1D) physics [4,18]. As a recent example, $\mathrm{YSi}_{2}$ nanowires with cross sections as small as $0.5 \mathrm{~nm}^{2}$ were shown to reveal van Hove singularities, a clear benchmark for a 1D electronic structure. Short-range charge

*tegenkamp@fkp.uni-hannover.de order along these 1D wires was found coming along with an opening of an electronic gap, however, the Peierls model is inappropriate and details about the electron-phonon coupling are still under debate [4]. Latest temperature-dependent transport measurements on these nanowires indicate a thermally assisted tunneling of polarons showing that quantum transport suffers from atomic-scale charge trapping defects [5].

The most prominent rare earth silicide nanowire systems are grown on $\mathrm{Si}(100)$ surfaces [19-23]. Often a bunching is observed where each individual nanowire is $2-3 \mathrm{~nm}$ wide. Their lengths may reach several $100 \mathrm{~nm}$, in some cases even above $1 \mu \mathrm{m}$, but usually substrate steps limit the length growth. These nanowires are characterized by a metallic band structure and there are indications for a one-dimensional dispersion [20,21]. While much effort was spent on growth, only a few transport studies were performed to date $[5,6]$.

The transport properties in such thin wires are severely influenced by atomic-scale defects [6] and finite size effects [24]. Intensive theoretical effort was made to understand the effect of an increased surface to volume ratio in nanostructures coming along with the so-called resistivity size effects first observed by Thomson [25]. The pioneering work by Fuchs and Sondheimer addressed the impact of diffusive surface scattering of electrons by introducing a specularity parameter $p$ [26,27]. Since then manifold descriptions of the transport process in more realistic geometries were developed both on semiclassical and quantum based models [28-30]. As we will show below, a quantitative description on an atomic level succeeds if both surface and bulk transport channels as well as details of the atomic-scale roughness are considered. An instrument-based breakthrough was the recent development of multiprobe scanning tunneling microscopy (STM) systems, which allow one to perform in situ transport measurements and high resolution STM studies of the same nanowire. 
In this work, we present a comprehensive study of the transport properties of individual $\mathrm{TbSi}_{2}$ nanowires grown on vicinal $\mathrm{Si}(001)$ substrates. Thereby, we concentrate first on the growth and atomic structure. Scanning tunneling spectroscopy (STS) revealed that the wires are electronically decoupled from the Si substrate in agreement with density functional theory (DFT) calculations. In the second part we explain in detail how to perform in situ transport measurements on single nanowires. In contrast to previous studies [5,6], we are able to make ohmic contacts directly using the STM tips without any need of further support contacts. Depending on the width and height of the wires, characterized in detail by STM, we finally determined the resistivities. The observed size dependence of the resistivity will be conclusively explained in the framework of the Chatterjee-Meyerovich [31] model assuming coherence effects between surface and bulk transport channels. Moreover, the roughness parameters deduced from the data analysis agree very well with those determined from the STM analysis.

\section{EXPERIMENT AND COMPUTATIONAL METHODS}

Tb silicide nanowires were formed by self-assembly on $\mathrm{Si}(001)$ wafers with a nominal miscut of $4^{\circ}$ towards the [110] direction and a bulk resistivity $\rho$ of $1-20 \Omega \mathrm{cm}$ ( $p$-doped with B). The substrates were first degreased subsequently for $10 \mathrm{~min}$ in an ultrasonic bath of acetone and isopropyl alcohol, then rinsed in deionized water and dried under pure $\mathrm{N}_{2}$. Finally, they were outgassed in ultrahigh vacuum by direct-current (dc) heating at $700^{\circ} \mathrm{C}$ for several hours and subsequent flash annealing cycles up to $1150^{\circ} \mathrm{C}$ for around $10 \mathrm{~s}$ while keeping the pressure below $2 \times 10^{-9}$ mbar. The temperature was controlled by an infrared pyrometer (LUMASENSE IG 140 , accuracy in probed temperature region $\pm 5^{\circ} \mathrm{C}$ ) and the emissivity coefficient $\epsilon$ was calibrated in feedback controlled manner with respect to a Si substrate. This cleaning procedure allows one to fully desorb $\mathrm{Si}$ oxides and carbon contaminants from the surface yielding $2 \times 1$ reconstructed terraces [32] separated by double-atom height steps in consistency with low energy electron diffraction spot analysis (not reported here).

$\mathrm{Tb}$ was evaporated on the vicinal $\mathrm{Si}(001) 2 \times 1$ surfaces heated at $700^{\circ} \mathrm{C}$ via a homebuilt electron-beam source and the deposited amount was controlled by a calibrated quartz-crystal microbalance. $\mathrm{Tb}$ silicide wires were grown by depositing around 0.8 monolayers $(\mathrm{ML})$ of $\mathrm{Tb}(1 \mathrm{ML}=\mathrm{Si}$ atomic surface density of $6.8 \times 10^{14}$ atoms $/ \mathrm{cm}^{2}$ ). After Tb deposition, the dc heating of the sample was immediately turned off in order to reduce the competitive growth of islands on the Si surface (cf. Fig. 1), as reported before for other RE metals $[33,34]$.

The morphology and dimensions of the as-grown wires were studied by both scanning electron microscopy (SEM) and STM investigations. Typically, a primary electron beam acceleration voltage of $15 \mathrm{kV}$ and an emission current of $1.0 \mathrm{nA}$ were employed for all the SEM images reported here, while the STM images were taken in constant current mode with a sample bias voltage $\left(V_{\mathrm{B}}\right)$ of $+2 \mathrm{~V}$ and tunneling currents $\left(I_{\mathrm{T}}\right)$ between 20 and $100 \mathrm{pA}$. Finally, two-point-probe transport measurements were performed on both the wires and the substrate using the capabilities of our multiprobe STM/SEM system (Omicron, Nanoprobe), which is equipped with three low-resolution probes specifically designed for

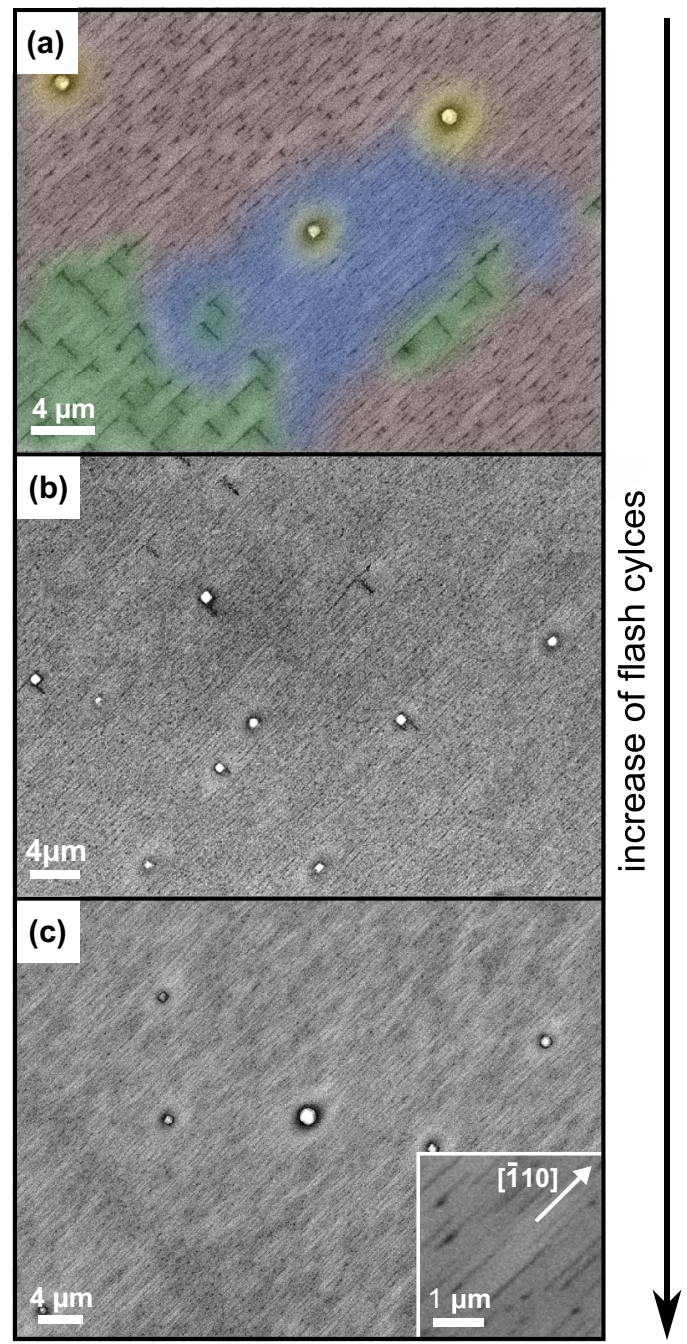

FIG. 1. SEM micrographs (plan-view) of $\mathrm{TbSi}_{2}$ wires grown on a $\mathrm{Si}(001)-[110] 4^{\circ}$ for different flash annealing cycles of the $\mathrm{Si}$ sample. (a) Three different nanostructure types are initially visible: $\mathrm{TbSi}_{2}$ wires aligned along the [110] direction (highlighted in red, blue), most likely $\mathrm{Tb}$ silicide islands (highlighted in yellow), and apparently bigger $\mathrm{TbSi}_{2}$ wires sometimes rotated by $90^{\circ}$ (in green). (b),(c) Further flash annealing of the $\mathrm{Si}(001)$ substrate (up to 100 cycles) generally results in samples that only show the thinnest wires [highlighted in blue in (a)].

transport analysis on nanometer-scale, one high-resolution probe for classical STM topography and an SEM column for the precise positioning of the contacting tips. Homemade $\mathrm{NaOH}$ etched $\mathrm{W}$ wires are used as STM tips.

DFT electronic-structure and total-energy calculations were performed to investigate the structural and electronic properties of the wires, and to compare them with STM/STS data. The calculations were performed with the VASP simulation package [35-37], within the Perdew-Burke-Ernzerhof formulation [38] of the generalized gradient approximation [39] (GGA-PBE). In particular, we model the silicide wires with slabs consisting of $8 \mathrm{Si}$ substrate layers decoupled by about $30 \AA$ of vacuum. $\mathrm{H}$ atoms saturate the dangling bonds at the lower side of the slab. The structures are optimized until the residual Hellmann-Feynman forces are lower than $0.01 \mathrm{eV} / \AA$. 
The lowest four $\mathrm{Si}$ atomic layers and the $\mathrm{H}$ atoms are kept constrained in order to model the substrate. A $4 \times 12 \times 1$ Monkhorst-Pack [40] k-point mesh was used to carry out the integration in the Brillouin zone for structural optimization. $a b$ initio thermodynamics was used to determine the most favorable wire geometry among several models that were derived from the measured STM data. In order to calculate numerically converged the site projected local density of states (LDOS) the k-point sampling has been increased, and a $4 \times 16 \times 1 \mathbf{k}$-point mesh was used. For the comparison with the differential conductivity measurements, the LDOS of the two outer atomic layers are employed.

\section{RESULTS AND DISCUSSION}

\section{A. Formation of $\mathrm{TbSi}_{2}$ wires on $\mathrm{Si}(001)$ substrates}

The growth process of $\mathrm{TbSi}_{2}$ wires by adsorption of $\mathrm{Tb}$ on planar as well as vicinal $\mathrm{Si}(001)$ surfaces was recently investigated in detail in Ref. [41]. The self-assembly of nanometer-sized silicide wires on $\mathrm{Si}$ proceeds in two steps: first, Tb induces a $2 \times 7$ reconstructed wetting layer on planar $\mathrm{Si}(001)$ for coverages of about $0.4 \mathrm{ML}$, while subunits of this reconstruction are found on vicinal $\mathrm{Si}(001)$ [41]. Any supplementary $\mathrm{Tb}$ deposition leads to the epitaxial growth of $\mathrm{TbSi}_{2}$ nanowires preferentially along the [110] direction, i.e., perpendicular to the $\mathrm{Si}$ dimer rows of the $2 \times 1$ reconstruction [41,42], until a step edge is reached. Depending on the annealing temperatures and $\mathrm{Tb}$ coverage rates, the growth of single or even bundled nanowires is promoted [41]. If not stated otherwise we use for both structure types the term nanowire. Also, a $2 \times 7$ reconstruction was found at low coverages $(\leqslant 0.5 \mathrm{ML})$ for other RE metals (Gd [43], Dy [44], Ho [45]), behaving as a precursor to the nanowire growth [45] and tending to fill the bare substrate between silicide wires at higher coverages $(\approx 1 \mathrm{ML})$.

\section{Growth and structure of wires}

Figures 1(a)-1(c) show SEM images demonstrating the successful growth of $\mathrm{Tb}$ silicide nanowires on large areas of the vicinal $\mathrm{Si}(100)$ substrate. The wire length typically varies between 2 and $4 \mu \mathrm{m}$, while their maximum width is always smaller than $30 \mathrm{~nm}$ (see STM results below). Moreover, it is evident that the substrate miscut promotes wire growth along Si steps [cf. blue and red areas in Fig. 1(a)], corresponding to the [110] direction, apart from a few $\mathrm{Tb}$ silicide islands (highlighted in yellow) and bigger $\mathrm{TbSi}_{2}$ wires, which are oriented either along the [110] or the [110] direction (in green).

Noteworthy, the $\mathrm{Tb}$ silicide islands with an average diameter of $0.4-1 \mu \mathrm{m}$ behave as getters and show no wires in their surroundings. This effect has been already observed in various STM studies for different RE metals $[19,42,46]$ and is mainly related to high diffusion lengths of $\mathrm{Tb}$ at high temperatures. While a few $\mathrm{Tb}$ silicide islands are always present, the density of the wires, growing perpendicularly with respect to the main growth direction, reduces drastically with increasing the flash annealing cycles before $\mathrm{Tb}$ deposition, resulting finally in a homogeneously covered surface with apparently smaller wires [cf. Figs. 1(a) and 1(c)]. It is well known that extended dc heating severely modifies the step height and density on vicinal

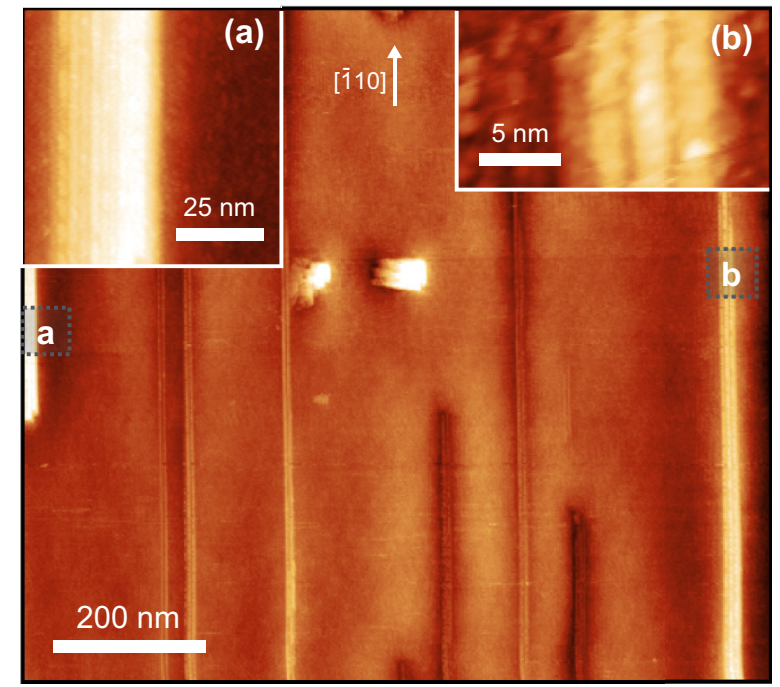

FIG. 2. STM overview image $\left(V_{\mathrm{B}}=2 V ; I_{\mathrm{T}}=100 p A\right)$ of $\mathrm{TbSi}_{2}$ wires grown on $2 \times 1 \mathrm{Si}(001)$ substrate after many $(>50)$ flashannealing cycles. The insets (a) and (b) show detailed STM images of the two $\mathrm{TbSi}_{2}$ wire bundles labeled with $a$ and $b$ in the figure.

Si substrates $[47,48]$ typically inducing large or even giant step bunching, which could drastically hinder the nanowire growth across the steps, as evident from the comparison of Figs. 1(a) and $1(\mathrm{c})$.

A high resolution STM image of a wire ensemble comparable to that of Fig. 1(c) is shown in Fig. 2. It is known that RE deposition promotes step movement and flattening of the Si substrate especially in the vicinity of the silicide wires $[19,34,41]$; thus step bunches are out of the scan range of the STM micrograph. As it is obvious from the insets in Fig. 2 the silicide wires exhibit a substructure, i.e., they consist of bundles of single wires separated by atomically thin grooves in agreement with results of a recent TEM study [49]. The widths of the single wires are quantized in units of the $\mathrm{Si}$ surface lattice constant along $a_{[110]}=$ $0.384 \mathrm{~nm}$ with the thinnest (broadest) being $3 a_{[110]}=1.15 \mathrm{~nm}$ $\left(8 a_{[110]}=3.07 \mathrm{~nm}\right)$ wide. The majority of the wires has an average width of $(6 \pm 1) a_{[110]}=(2.2 \pm 0.4) \mathrm{nm}$, which is in full agreement with previous STM studies [41,44]. In this study we concentrate exclusively on bundle structures. These bundles can be easily found by SEM, thus reliably contacted by the STM tips which enables us to investigate many wires.

\section{Surface roughness of the wires}

Besides the growth, we will briefly focus on a further important aspect. The STM image in Fig. 3(b) shows a wire bundle, which consists of five single wires, each with an average width of $2.5 \mathrm{~nm}$. The wire bundle has a maximum height of $0.9 \mathrm{~nm}$, roughly corresponding to three atomic steps (each $0.33 \mathrm{~nm}$ high [41]). However, only the first two layers form a compact structure, while the third one consists of an ensemble of $\mathrm{TbSi}_{2}$ nanoislands, which is responsible for the surface roughness and will have consequences for the transport properties.

The surface topography has been quantified by the so-called height-height correlation function (HHCF) $H(r)$ along the wire, which is defined as $H(r) \equiv\left\langle\left[h\left(r^{\prime}\right)-h\left(r+r^{\prime}\right)\right]^{2}\right\rangle$, where 
(a)

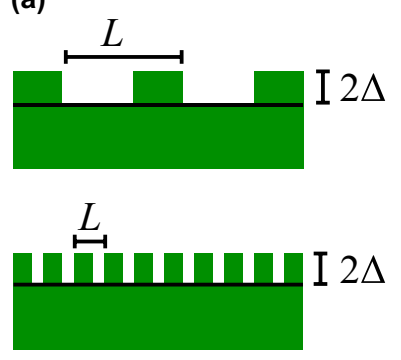

(b)

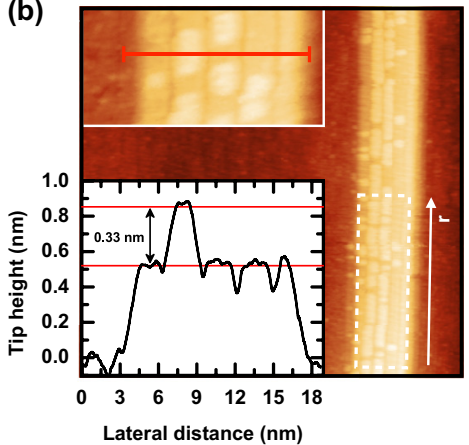

(c)

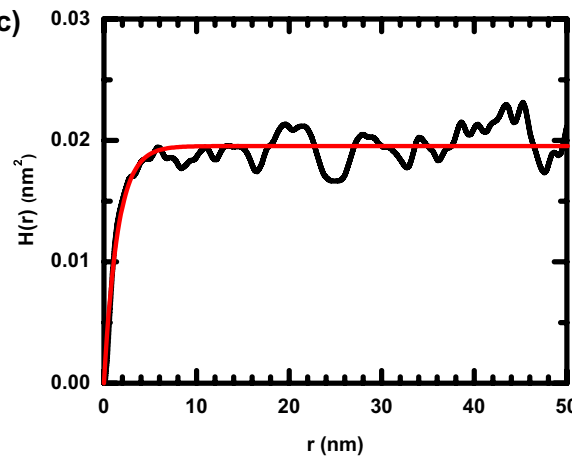

FIG. 3. (a) Two schematics to illustrate different correlation lengths $L$ and roughness $\Delta$. (b) High resolution STM image ( $V_{\mathrm{B}}=2 V$; $I_{\mathrm{T}}=100 \mathrm{pA}$ ) showing the formation of additional nanoislands on top of the wires. This is displayed in detail in the upper inset and analyzed quantitatively using the height contour shown in the lower inset. (c) Derived HHCF plot along the wire (black) with fit curve according to equation mentioned in the text.

$h(r)$ is the vertical position of the surface at lateral coordinate $r$ along the nanowire direction with respect to a reference plane, while the angular brackets $\langle\cdot\rangle$ denote the averaging. The HHCF is usually assumed to be exponential (or Gaussian) and expressed as a function of the surface root-mean-square roughness $\Delta$ and the lateral correlation length $L$ of the surface [50-52] [cf. schematics of Fig. 3(a) for reference]. Here, we adopt the exponential correlation, i.e., $H(r)=$ $2 \Delta^{2}(1-\exp (-r / L))$, which shows a better agreement to our experimental findings. From the best fit of the experimental $\mathrm{HHCF}$ [cf. red solid curve in Fig. 3(c) for reference], the values, which seem to be typical for our $\mathrm{TbSi}_{2}$ nanowires, are a surface roughness of $\Delta \approx 0.1 \mathrm{~nm}$ (almost matching the experimental nanowire surface roughness of $\pm 0.15 \mathrm{~nm}$ ) and lateral correlation length of $L \approx 1 \mathrm{~nm}$. We will show below that these values agree reasonably well with those determined from transport measurements.

\section{Electronic properties of wires}

As a consequence of the stress-mediated uniaxial growth and the silicide formation, the nanowires are in close contact with the Si surface. In the following, we show STS spectra for our $\mathrm{TbSi}_{2}$ wires [cf. Fig. 4(a)]. The spatially resolved STS spectra reveal that the central part of the wire bundle has no gap in contrast to the STS spectra taken on the host material. The $d I / d V$ spectra taken at a distance of about $5 \mathrm{~nm}$ from the wire shows basically the $\mathrm{Si}$ band gap. Please note that tip-induced band bending effects on these low-doped surfaces are not fully negligible, revealing easily an apparently larger band gap [53]. Most importantly, the $\mathrm{Tb}$ induced wetting layer between the wires is insulating, thus not influencing our transport measurements due to parasitic current paths. On the contrary, the band gap vanished in the center of a five-wire wide $\mathrm{TbSi}_{2}$ bundle. Its conductivity will be further investigated below via local transport measurements. Generally, these results for $\mathrm{TbSi}_{2}$ wires are in agreement with many studies performed to date on various $\mathrm{RESi}_{2}$ materials [21,54-57] using either STS or angle resolved photoelectron spectroscopy, which corroborate the presence of metallic states at room temperature.

In order to confirm the STS findings, we performed DFT calculations to determine the LDOS in different regions of the nanowire-decorated surface. The structural model of a stable wire geometry determined from $a b$ initio thermodynamics as well as the space regions representing the sectors defined as substrate, transition, and on wire are shown in Fig. 4(b). Thereby we average the LDOS laterally (i.e., across the wire axis), using an integration area that corresponds to the substrate lattice constant. The energy dependent LDOS, which was first derived by this approach for a clean surface, is shown in the left panel of Fig. 4(b). Additionally, in order to address the origin of these states, we also performed DFT calculations for hydrogenated surfaces where the surface dangling bonds of both the silicide wire as well as the $\mathrm{Si}(001)$ surface were saturated.

Compared to the substrate, a modification of the LDOS at the Fermi energy is observed in the transition region, in reasonable agreement with the experiment. A further remarkable increase of the DOS is observed in the on wire region, in close agreement with the measurements. As shown in Fig. 4(b), the removal of surface states due to $\mathrm{H}$ adsorption reduces substantially the LDOS in the transition region with respect to the clean surface, showing that it is largely related to surface localized states that arise from the rearrangement of the substrate due to the wire formation. $\mathrm{H}$ adsorption on the clean $\mathrm{Si}(001)$ substrate region leads, as expected, to a LDOS that is reminiscent of the Si bulk density of states. The metallicity of the nanowire itself, in contrast, is not affected by $\mathrm{H}$ adsorption. Its robustness with respect to surface termination effects is related to the existence of metallic states within the RE nanowire. The mapping (not shown) of the LDOS onto the different elements $(\mathrm{Si}, \mathrm{Tb}, \mathrm{H})$ reveals that the peaks (marked by dashed lines) reflect $\mathrm{Tb}$-Si hybrid states, which are responsible for the metallicity. Moreover, the LDOS calculated for a single $\mathrm{TbSi}_{2}$ wire is in almost perfect agreement with the $d I / d V$ spectrum taken on bundles. This demonstrates that width-dependent strain effects are of minor importance. As shown by a recent TEM investigation, the bundle structure consists of single nanowires as a consequence of the stress developing during growth along the [110] direction [49].

\section{B. Transport properties of $\mathbf{T b S i}_{2}$ wires}

\section{Ohmic contacts via STM tips approached onto single $\mathrm{TbSi}_{2}$ wires}

The electrical transport properties of the $\mathrm{TbSi}_{2}$ wires described above were further investigated in situ (without 

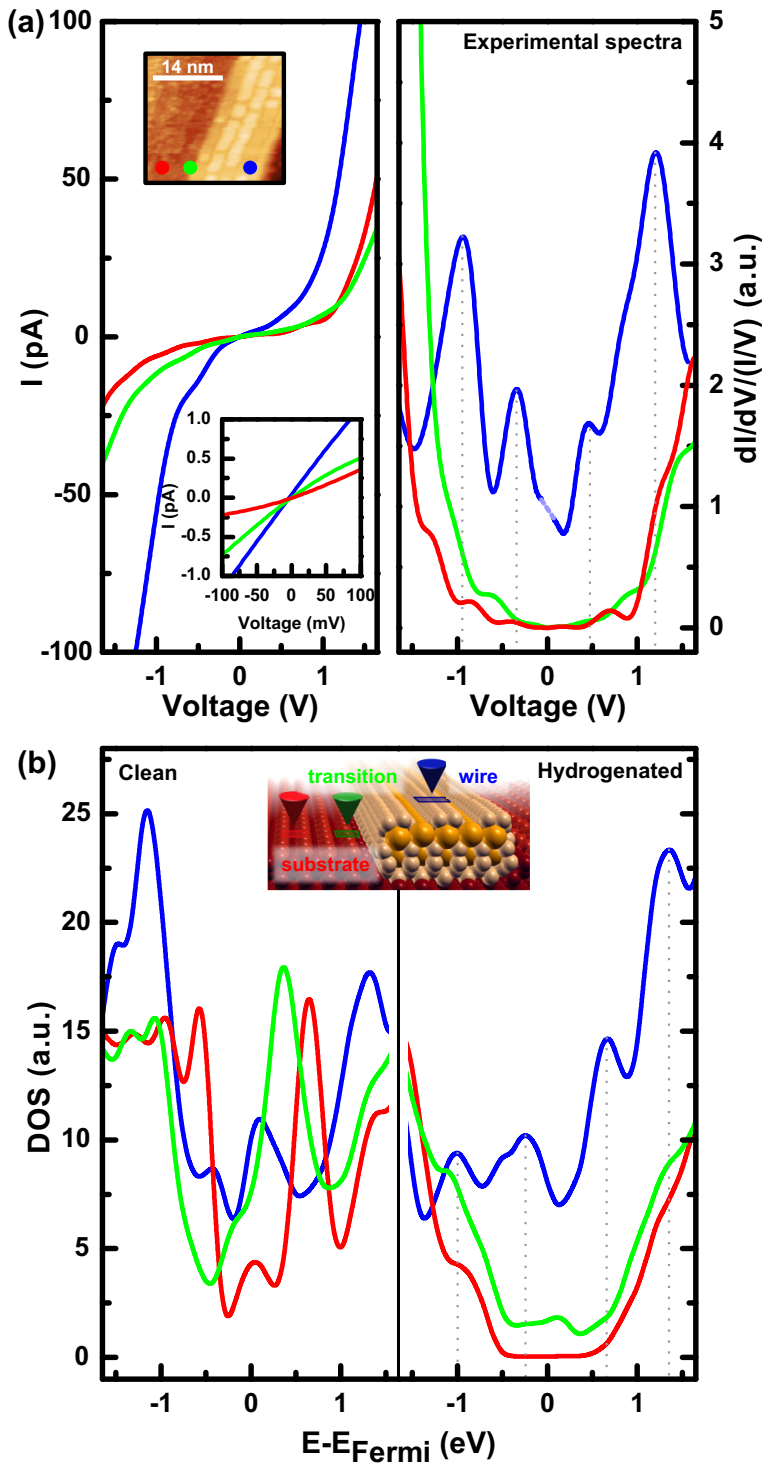

FIG. 4. (a) Experimental $I(V)$ (left) and normalized $(d I / d V) /(I / V)$ spectra (right) taken at respective color-coded positions indicated in the STM image in the inset. The color code of the spectra corresponds to the one shown in the inset. The metallic behavior of the $\mathrm{TbSi}_{2}$ wires is obvious from both the steep $I(V)$ characteristics (see magnification in inset) and the finite $(d I / d V) /(I / V)$ signal at the Fermi energy. (b) The theoretical LDOS in the different space regions of the surface, labeled as substrate, transition, and wire in the inset. The LDOS for a fully $\mathrm{H}$-saturated surface structure (right panel) is in excellent agreement with the experiment. For further details see text.

breaking the vacuum) using the multiprobe capabilities of the STM system. Thereby, special attention was paid for a gentle approach of the tips in order to avoid, or at least to reduce, damages upon contacting the silicide wires. The approaching procedure is illustrated by the sequence of SEM images shown in Fig. 5. First, the STM tips were brought into the tunneling regime $\left(V_{B}=-2 \mathrm{~V} ; I_{T}=500 \mathrm{pA}\right)$ on the free substrate in between the wires [cf. Fig. 5(a)]. After turning off the STM feedback control, the tip is retracted by around $50 \mathrm{~nm}$, navigated to the desired position above the wire and then

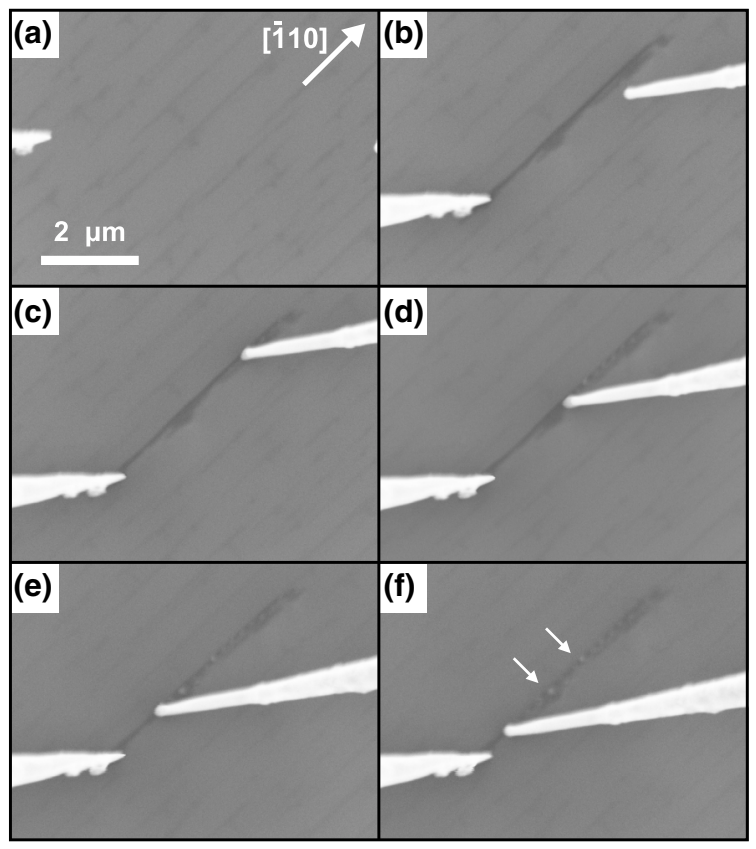

FIG. 5. Sequence of plan-view SEM micrographs depicting the contacting procedure of a single $5 \mu \mathrm{m}$ long $\mathrm{TbSi}_{2}$ wire with two STM probes. (a)-(c) First, the two tips are placed at the ends of the wire, (d)-(e) then keeping fixed the position of one tip, the second one is moved step-by-step towards the first, performing at each position an $I(V)$ measurement in order to determine the wire resistance. (f) Despite the adopted extremely gentle measurement procedure, white spots (marked by arrows) are appearing on the wire and indicate a slight local damage.

slowly lowered towards the wire surface in steps of $0.1 \mathrm{~nm}$. As soon as a stable contact between a wire and the tip is reached, the wire appears darker in the SEM image [cf. Fig. 5(b)] caused by the modified surface potential. The homogeneity of the contrast along the wire is indicative for its conductivity, while the unchanged contrast of the surrounding substrate indicates a good insulation from the nanowire. Since the SEM contrast remains unchanged by contacting the wire with a second tip, well-defined probe distant dependent measurements become at first feasible. The precise positioning of the second tip at various lateral positions is achieved by biasing the approaching tip with respect to the first one at around $0.5 \mathrm{~V}$. Finally, reliable contacts are realized as soon as the measured current jumps from $10^{-10} \mathrm{~A}$ (i.e., the noise level with blanked SEM beam) to about $10^{-7} \mathrm{~A}$.

Figure 6 shows two-point-probe $I(V)$ curves for a $\mathrm{TbSi}_{2}$ wire at different probe spacings. All $I(V)$ curves reveal a linear characteristics as expected for ohmic contacts between the $\mathrm{W}$ tips and a metallic $\mathrm{TbSi}_{2}$ wire. Moreover, the resistance clearly decreases as the probe spacing between the tips is decreased. For the sake of comparison, we also show in the inset (a) in Fig. 6 the $I(V)$ curve when one of the tips accidently contacts the substrate. Besides a Schottky-type characteristics, the current is about two orders of magnitude smaller than that on the wire for comparable voltages. As mentioned already above, this result directly confirms the insulating nature of the wetting layer covering the bare $\mathrm{Si}(001)$ substrate between the wires as well as a sufficiently high Schottky-barrier height to 


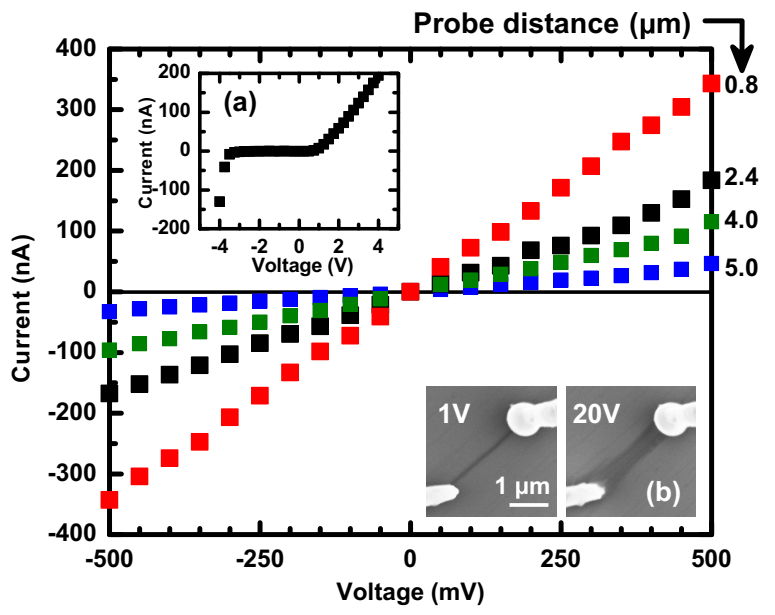

FIG. 6. Two-point-probe $I(V)$ curves of the same $\mathrm{TbSi}_{2}$ wire shown in Fig. 5 for various probe spacings. The different slopes reflect the linear decrease of resistance as the two probes get closer. For sake of comparison, inset (a) shows an $I(V)$ curve when one of the tip accidently contacts the substrate, while inset (b) shows the wire-desorption induced by Joule heating after applying an extremely high voltage of $20 \mathrm{~V}$.

the substrate, suggesting that all the current flows exclusively through the silicide wire (i.e., no significant current paths along the Si substrate). This is further corroborated by inset (b) in the same figure showing a $\mathrm{TbSi}_{2}$ wire contacted by two STM probes before (left) and after (right) applying a voltage of $20 \mathrm{~V}$. The Joule heating induced by high-current densities $\left(>10^{8} \mathrm{~A} / \mathrm{cm}^{2}\right.$ ) preferentially flowing through the wire causes its complete and selective desorption as well as some modifications of the neighboring substrate surface, e.g., by electromigration or by local desorption of the wetting layer.

Making ohmic contacts deals inevitably with a mechanical impact. As evident from Fig. 5(f) by the white spots visible on top of the wires, the $\mathrm{TbSi}_{2}$ nanowires show morphological changes upon contacting with the probes. Moreover, the wire resistance is increased if a previously contacted area is between the two contacting probes, which is again indicative for a local wire damage. Therefore, the transport measurements shown here were all performed starting with largest probe spacings. Because of this technical limitation, collinear four-point-probe measurements, usually recommended in order to rule out contact-resistance contributions, were not performed here in favor of two-point-probe measurements with variable probe spacings. As we will show below, the contact resistances in our case are sufficiently small. We also like to emphasize that the white spots visible in Fig. 5 are more or less of the same contrast, i.e., the positioning of our probes with contact sizes in the order of the wire width is a reliable and very reproducible procedure.

\section{Two-point-probe profile analysis of $\mathrm{TbSi}_{2}$ wires}

Figure 7 shows now the typical two-point-probe resistance of a $\mathrm{TbSi}_{2}$ nanowire versus the probe spacing. The impedance of the wire increases linearly with the probe spacing, suggesting a classical 1D diffusive transport along the silicide wires [58]. Interestingly, at around $3.5 \mu \mathrm{m}$ the slope changes

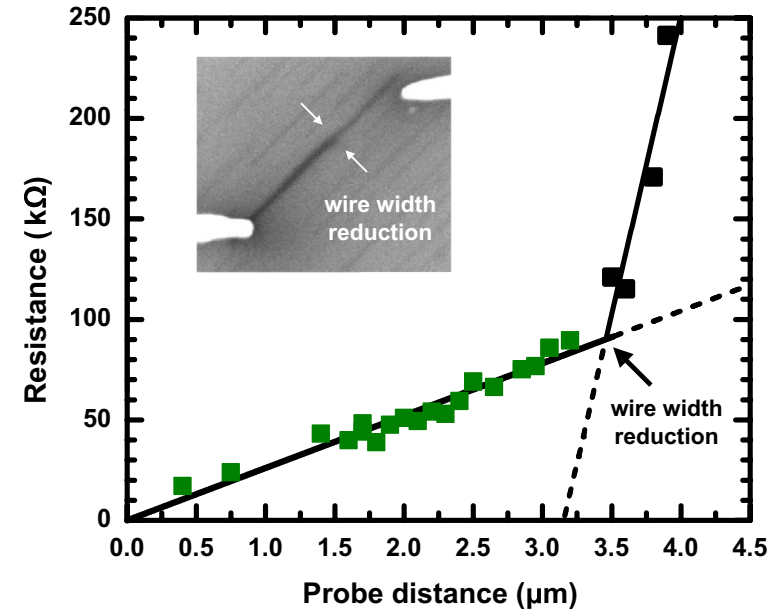

FIG. 7. Two-point-probe resistance vs probe spacing of a $\mathrm{TbSi}_{2}$ nanowire. The resistance increases linearly with the probe distance as expected for diffusive 1D transport. Moreover, the sudden change of the slope at around $3.5 \mu \mathrm{m}$ can be attributed to a reduction of wire size (structural inhomogeneity) indicated in the inset.

drastically. Close inspection of the SEM image (see inset) reveals at this point a reduction in the wire size, which turns out to be responsible for the sudden change of the resistance. The two solid curves in Fig. 7 are two linear fits of the experimental data before and after the wire size reduction, respectively. Noteworthy, the solid curve fitted in the regime without the defect intercepts the origin of the axes, which indicates an almost negligible contact resistance at both wire-tip interfaces. The resistance per length of the silicide wire is around $26 \mathrm{k} \Omega / \mu \mathrm{m}$, which increases to $300 \mathrm{k} \Omega / \mu \mathrm{m}$ when the region with the reduced wire size in this particular wire is probed.

Being aware of these pitfalls, we performed two-pointprobe transport measurements on almost fifty (homogeneous) wires of different size and length on several samples, which were prepared with different numbers of initial annealing cycles (cf. Fig. 1). Please note that only those $\mathrm{TbSi}_{2}$ wires with a constant width along their entire length are considered in the following. Classically, the resistance $R$ of the wire as a function of the probe spacing $s$ is $R(s)=\rho s A^{-1}$, where $\rho$ is the wire resistivity and $A$ its cross-sectional area. In a double logarithmic plot of $R$ versus $s$, as shown in Fig. 8, an identical slope results for different wires, while only the height of the curves varies as a function of the wire resistivity and/or cross section. Different symbols in Fig. 8 refer to distinct wires, but same colors are used for wires with a comparable resistance per length. Roughly, three classes of wires, labeled by $w 1, w 2$, and $w 3$, can be identified and are highlighted in blue, red, and green with a resistance per length of approximately $1,0.2$, and $0.03 \mathrm{M} \Omega / \mu \mathrm{m}$, respectively.

The formation of the different types of wires depends on the preparation and atomistic details of the $\mathrm{Si}$ substrate. For instance, wire types $w 1$ and $w 2$ are typically observed on samples, which were flash annealed more than 50 times [cf. Fig. 1(c)], while $w 3$ refers to the big wires, visible in Figs. 1(a) and 1(b), that are exclusively oriented along the [110] direction. As we will show below, the $w 1$ - and w2-type wires differ mainly with respect to their widths. Slightly modified 


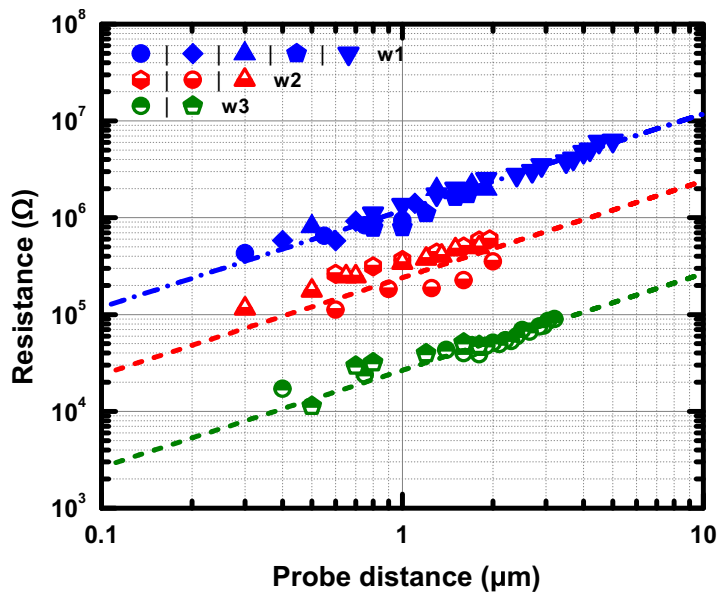

FIG. 8. Resistance determined by a two-point-probe geometry for several wires of different lengths and sizes. Three wire classes, named $w 1, w 2$, and $w 3$ with a resistance per length around $1,0.2$, and $0.03 \mathrm{M} \Omega / \mu \mathrm{m}$, are highlighted in blue, red, and green, respectively.

preparation parameters should result in the formation of wires with intermediate widths (between $w 1$ - and $w 2$-type). In this sense, the nomenclature is rather artificial and accounts only for the findings shown in Fig. 8.

The large range of measured wire resistances can be ascribed to characteristic structural motifs, e.g., to the wire cross section $A$. Therefore, in order to make a close connection of the transport properties with the atomic structure, we performed for some wires also high-resolution STM measurements [the setup is sketched in the SEM image in Fig. 9(a)]. First, two low resolution STM tips were placed at the ends of a wire and the type of wire, i.e., $w 1, w 2$, or $w 3$, was deduced from an $I(V)$ measurement. Thereafter, the high resolution STM tip was navigated between the probes to measure locally the topography with high resolution, as shown, e.g., for a w1- and w2-type wire in Figs. 9(c) and 9(d), respectively. Figure 9(b) shows a STM scan, which exactly corresponds to the SEM image shown in Fig. 9(a). Apparently, four scratches (highlighted by white dashed ellipses) at the ends of the two wires marked with $w 1$ and $w 2$ in Figs. 9(a) and 9(b) result from the ohmic contacts of the low resolution STM tips. Finally, the high resolution STM tip was retracted and additional $R(s)$ measurements along the wire profile were performed in order to further rule out effects of wire inhomogeneities.

The wires marked by $w 1$ and $w 2$ in Figs. 9(a) and 9(b), respectively, show a resistance per length of $940 \mathrm{k} \Omega / \mu \mathrm{m}$ and $480 \mathrm{k} \Omega / \mu \mathrm{m}$, respectively. Both types of wires have a similar height (between two and three silicide layers, i.e., $0.66 \mathrm{~nm}$ and $0.99 \mathrm{~nm}$ ). However, since only the second layer appears entirely continuous in the STM images, an effective height of $0.66 \mathrm{~nm}$ is assumed here [corresponding to the red lines in the line scans in Figs. 9(c) and 9(d)]. Indeed, better resolved STM images (cf. Fig. 3 for reference) show an incomplete coverage for the third layer along the wire axis, which does not contribute to the wire current flow while it is mainly responsible for the surface roughness of the wire. The full width at half of the effective height of wires $w 1$ and $w 2$ is around $18 \mathrm{~nm}$ and $33 \mathrm{~nm}$, respectively, as indicated by the dashed lines in the line
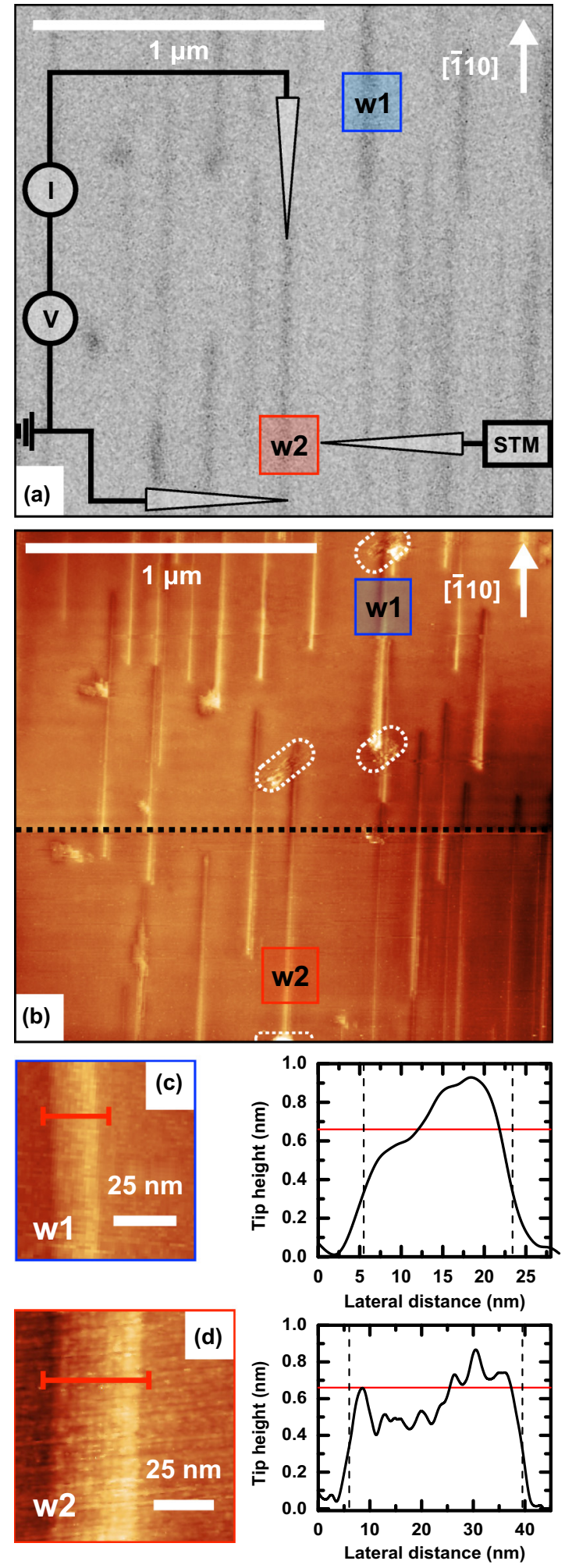

FIG. 9. SEM (a) and STM (b) micrographs showing the same surface area. The schematic drawing in (a) shows how both transport studies as well as STM images of the same wire were obtained. The STM image in (b) $\left(V_{\mathrm{B}}=2 \mathrm{~V} ; I_{\mathrm{T}}=100 \mathrm{pA}\right)$ is composed by two micrographs connected at the black dashed line. The areas marked by dashed white ellipses show defects induced by contacting procedure for transport measurements. Red and blue framed squares highlight the area shown at higher magnification in (c) and (d), respectively. Red lines mark where line profiles were taken shown at the right. 


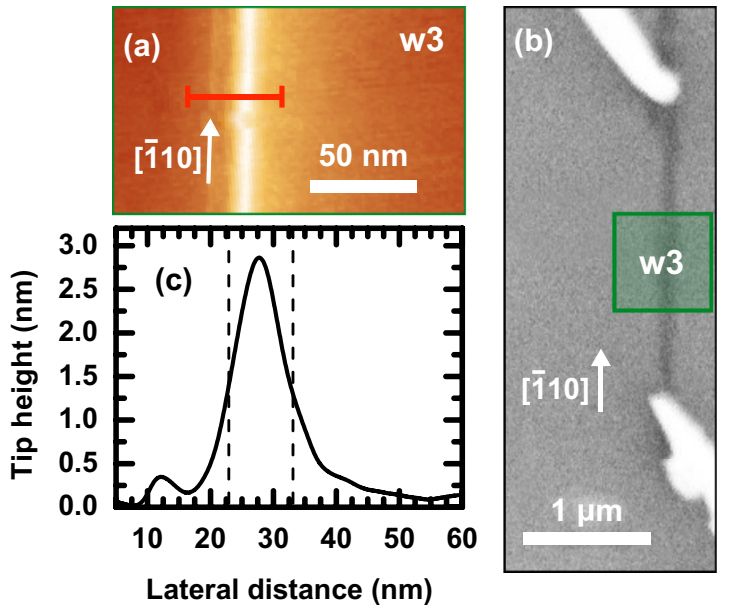

FIG. 10. (a) STM topography $\left(V_{B}=2 \mathrm{~V} ; I_{T}=100 \mathrm{pA}\right)$ of an apparently bigger wire. (b) SEM image showing the two-point-probe setup for such a wire. The color code corresponds to the one used in Fig. 8. (c) Height profile across the wire taken along the red line shown in (a).

profiles in (c) and (d). For both the $w 1$ - and w2-type wires the corresponding resistivity thus results to around $1100 \mu \Omega \mathrm{cm}$, which is roughly one order of magnitude larger than the value reported for a $12 \mathrm{~nm}$ thick $\mathrm{TbSi}_{2}$ film grown on a $\mathrm{Si}(111)$ substrate [59].

As mentioned in the context of Fig. 8, we found also socalled w3-type wires oriented along the [110] direction. The STM topography as well as a height profile across such a wire is shown in Fig. 10 and yields a width of around $10 \mathrm{~nm}$ and height of almost $3 \mathrm{~nm}$. The resistance per length of this wire amounts to $130 \mathrm{k} \Omega / \mu \mathrm{m}$ and refers to a resistivity of $380 \mu \Omega \mathrm{cm}$. The latter value is closer to the one expected for a compact thick $\mathrm{TbSi}_{2}$ film [59], suggesting a height-dependent increase for the nanowire resistivity while decreasing the wire size.

\section{Resistivity size effects in $\mathrm{TbSi}_{2}$ wires}

As just shown, the structurally different wires exhibit different resistivities. For a quantitative treatment of this interesting phenomenon we have correlated their widths and heights to their resistance per length. Exemplarily, we have characterized seven of these wires in detail by STM (see Table I for reference). The lateral width of the wires varies between $10 \mathrm{~nm}$ and $110 \mathrm{~nm}$, while the apparent height ranges from
$0.67 \mathrm{~nm}$ to $9 \mathrm{~nm}$. Finally, with these structural parameters the resistivities were calculated and plotted versus their apparent height in Fig. 11. Noteworthy, the wire resistivity has no clear dependence on the wire width or cross section, while it strongly decreases with increasing wire height. In the following, different transport models are discussed and compared quantitatively to our experimental findings.

\section{Modeling of the resistivity in nanowires}

Nanosized materials often exhibit larger electrical resistivities compared to their macroscopic counterparts due to the additional scattering of the conduction electrons at the material surfaces and/or grain boundaries [25,26,28,29,60-63]. This effect is dominant if the electron mean free path $\ell$ becomes comparable to the extension of the nanoobject. For instance, a free electron density and a bulk resistivity of $n=10^{22} \mathrm{~cm}^{-3}$ and $\rho_{\text {Bulk }}=90 \mu \Omega \mathrm{cm}$ for $\mathrm{TbSi}_{2}$ at room temperature [59] yields a mean free path of around $3 \mathrm{~nm}$ [27]. Similar values were reported for other silicides (e.g., $\mathrm{CoSi}_{2}$ [64-66] or NiSi [67]). Qualitatively, this already explains why the resistivity measured in our case is so sensitive to the height rather than to the width of the wires. Contributions to the material resistivity originating from grain boundaries, which are usually described in the framework of a classical theory by Mayadas $[63,68]$, are not considered here further, since high-resolution TEM investigations [69,70] reveal that the density of these bulk defects compared to the surface roughness is extremely low.

The apparent width of the investigated wires is larger than their electronic elastic mean free path. However, as mentioned above, the nanowires reveal a substructure. From a recent TEM study it turns out that they consist of single wires with an average width of only $2.2 \mathrm{~nm}$, which are separated from each other by atomically thin $(\approx 0.3 \mathrm{~nm})$ grooves [49]. The grooves between these subunits are atomically smooth $\left(\Delta_{\text {lateral }}=0\right)$, as seen from STM. Therefore, as we will show below, the resistivity of the wires is not affected by their limited width. Moreover, the radius of the current probes is always larger than the apparent width of the wires, so that all nanowires in a bundle are contacted. This justifies our approach to evaluate the transport data in terms of an effective width.

\section{Classical description of resistivity size effects}

Within a classical approach the transport phenomena in nanosized structures were considered first by Fuchs [26]

TABLE I. Structural parameters and transport properties of seven different wires. The resistivities are plotted in Fig. 11 as a function of wire height and can be reliable modeled within the Chatterjee-Meyerovich model. The error bars of the resistivities are calculated assuming an uncertainty of one atomic step for the $\mathrm{TbSi}_{2}$ nanowire height.

\begin{tabular}{lccllr}
\hline \hline Data point & Height $(\mathrm{nm})$ & Length $(\mu \mathrm{m})$ & Width $(\mathrm{nm})$ & Resistance $(\mathrm{k} \Omega)$ & Resistivity $(\times 100 \mu \Omega \mathrm{cm})$ \\
\hline 1 & 0.67 & 1.4 & 18 & 1300 & $11.2 \pm 5.6$ \\
2 & 0.67 & 1.0 & 34 & 482 & $10.7 \pm 5.4$ \\
3 & 1.2 & 1.0 & 17 & 402 & $8.0 \pm 2.2$ \\
4 & 2.0 & 1.0 & 50 & 52 & $5.2 \pm 1.7$ \\
5 & 3.0 & 2.1 & 10 & 271 & $3.8 \pm 0.8$ \\
6 & 4.0 & 3.2 & 25 & 40 & $2.8 \pm 0.5$ \\
7 & 9.0 & 2.9 & 110 & 4 & $1.5 \pm 0.1$ \\
\hline \hline
\end{tabular}




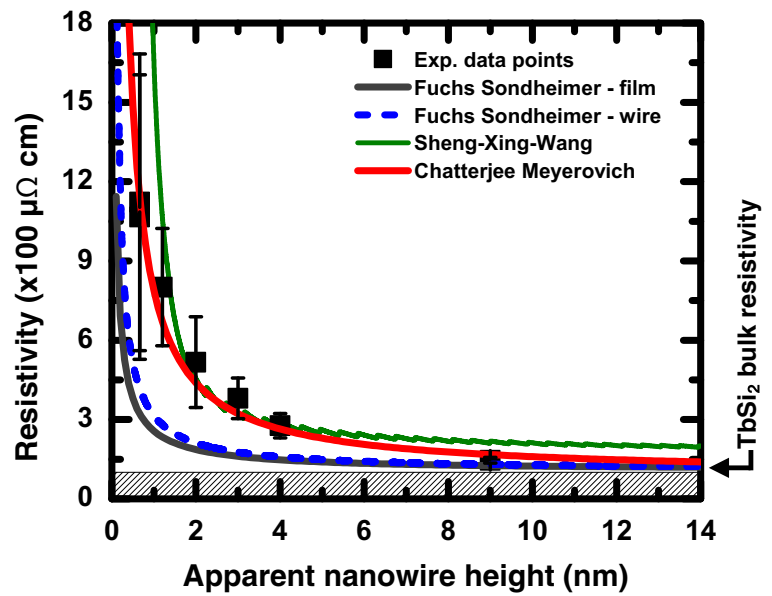

FIG. 11. Calculated resistivity vs the apparent height of the $\mathrm{TbSi}_{2}$ nanowires revealing a strong decrease with increasing height. The solid and dashed lines are deduced from classical and quantum theories. For details see text.

introducing a specular parameter $p$, i.e., the fraction $(1-p)$ of electrons scatters diffusively at the surfaces and interfaces, thus reducing the mean free path and, consequently, increasing the resistivity. Analytically, the ratio of the resistivities between bulk and film of thickness $D$ is expressed as [60]

$$
\begin{aligned}
\frac{\rho_{\text {bulk }}}{\rho_{\text {film }}}= & 1-\frac{3 \ell}{2 D}(1-p) \int_{0}^{\pi / 2} \cos \theta \sin ^{3} \theta \\
& \times \frac{1-\exp \left(-\frac{D}{\ell \cos \theta}\right)}{1-p \exp \left(-\frac{D}{\ell \cos \theta}\right)} d \theta .
\end{aligned}
$$

In Fig. 11 the solid black curve is based on this description, which cannot explain the experimental finding for small heights at all. Thereby, the worst case with $p=0$ was already assumed. Moreover, also the consideration of realistic geometries, i.e., wires instead of films [28,29,60,62,71], cannot explain our discrepancy. For instance, the blue dashed line is the result based on a theory by Moraga et al. [62] for wires with a rectangular cross section and a maximum width-to-height ratio of $\mu_{\max } \approx 3$, discussed in the context of Fig. 10. However, in most cases $\mathrm{TbSi}_{2}$ wires with $\mu>10$ are found (cf. Fig. 9), i.e., the values match almost the description of Fuchs [60], which can describe our data only qualitatively, in agreement with many other studies [72-77].

The Fuchs theory is based on the classical Boltzmann equation and relies on the model of a nearly free electron gas. Most severely, it does not explicitly include surface roughness and adopts a $p$-parameter independent of the electron energy and of the angle of incidence to the surface. We will see in the following that the surface morphology, parametrized by the rms roughness $\Delta$ and the correlation length $L$, indeed is the key characteristic for a quantitative description. Technically, the angular dependency of the $p$ parameter can be tentatively considered [78]. However, in our system the Fermi wavelength $\lambda_{F}$ is comparable to the surface roughness, which causes a fully diffusive scattering behavior, i.e., $p=0$.

\section{Quantum models for resistivity size effects including surface roughness}

More realistic models are based on a quantum mechanical description considering details of the surface topography, i.e., roughness $\Delta$ and correlation length $L$, as well as potential effects of electronic confinement [30,31,77,79-81]. The limitation of these theories is that they were solved for thin films rather than for wires. However, as seen in the context of the classical description, lateral constrictions only marginally affect the resistivity values. Here, we restrict ourselves to the theories elaborated by Sheng-Xing-Wang [30] (in the following SXW model) and the Chatterjee-Meyerovich [31] model (CM model).

The SXW model, based on the Kubo formalism in real space [82], considers both bulk and surface scattering. While for $D \gg \ell$ the model transforms into the Fuchs model [Eq. (1) $\}$, it almost reproduces for $D \ll \ell$ the description of Trivedi et al. [77] and the resistivity of the film can be expressed by

$$
\begin{aligned}
& \rho_{\text {film }}(D) \\
& =\left(\frac{2 e^{2}}{\lambda_{F} h k(D)} \sum_{n=1}^{n_{c}(D)} \frac{1-n^{2} / k(D)^{2}}{\frac{2 n_{c}(D)+1}{\frac{2 \pi}{\lambda_{F}} \ell k(D)}+\left(\frac{2 \pi^{2} L \Delta}{\lambda_{F} D}\right)^{2} \frac{S_{n_{c}}(D) n^{2}}{n_{c}(D)^{3} k(D)}}\right)^{-1}
\end{aligned}
$$

Thereby, $k(D)=2 D / \lambda_{F}, n_{c}(D)=\operatorname{Int}(k(D))$ denotes the number of occupied subbands, $\lambda_{F}$ is the Fermi wavelength, and $S_{n_{c}(D)}=n_{c}(D)\left(1+n_{c}(D)\right)\left(1+2 n_{c}(D)\right) / 6$. The first term in the denominator inside the summation of Eq. (2) represents the bulk scattering, while the second is responsible for the surface scattering [81] and is taken into account via the Mathiessen rule approximation. For the case that the bulk contribution is negligible, Eq. (2) reduces to the result reported by Fishman et al. [81]. Compared to the result obtained by Trivedi et al. [77] Eq. (2) is taking into account, besides surface roughness, lateral correlation effects.

In the context of the quantum model from ChatterjeeMeyerovich, considering the rough surface by an appropriate mapping onto an equivalent bulk Hamiltonian [83], the thin film resistivity is given by [31]

$$
\begin{aligned}
\rho_{\text {film }} \approx & \frac{m}{n e^{2}}\left[\frac{1}{\tau_{\text {tr }}^{b}}+\frac{16 \sqrt{2 \pi} E_{F}}{p_{F} \lambda_{F}} \frac{\Delta^{2}}{L d}\right. \\
& \left.+\frac{10 \sqrt{2} E_{F}}{\sqrt{\pi} p_{F} \lambda_{F}} \frac{\Delta^{2}}{L D}\left(\frac{\hbar}{2 \tau_{b} E_{F}} \frac{\lambda_{F}^{2}}{L^{2}}+\frac{\lambda_{F}^{2} m}{2 \tau_{b}^{2} E_{F}}\right)\right] .
\end{aligned}
$$

Here $\tau_{\mathrm{tr}}^{b}$ and $\tau_{b}$ denote the electron bulk transport and collision time, $m$ is the electron mass, and $E_{F}, p_{F}$, and $\lambda_{F}$ are the Fermi energy, momentum, and wavelength, respectively. Effectively, Eq. (3) should be considered as a semiclassical description, since the summation over the subbands was replaced by an integration of the density of states. The first terms represent the bulk and surface scattering, similar to the SXW model. In contrast, the remaining two terms describe the effect of interference between the surface and bulk transport channels. Indeed, there is clear evidence that the Mathiessen rule is violated in the proximity of mean free path thickness $[80,84]$ where the bulk and surface-scattering processes 
are not necessarily independent, but may strongly interfere with each other. Note that this contribution is inherently neglected in the SXW formalism, which adopts a white noise approximation (i.e., small correlation lengths). Both models were applied to the resistivity dependence shown in Fig. 11. We assumed a Fermi wavelength for $\mathrm{TbSi}_{2}$ material of $\lambda_{F}=$ $(8 \pi / 3 n)^{1 / 3} \approx 0.94 \mathrm{~nm}$, and an electron bulk transport time $\tau_{\mathrm{tr}}^{b}=\left(m / e^{2} n \rho_{\text {bulk }}\right) \approx 3 \times 10^{-15} \mathrm{~s}$, which is also comparable to the collision time $\tau_{b}$ since the Debye temperature of $\mathrm{TbSi}_{2}$ amounts to $\Theta_{D} \approx 260 K \leqslant 300 K$ [59]. The green solid curve in Fig. 11 reveals the best fit of the experimental data using Eq. (2) with a surface roughness of $\Delta_{S X W} \simeq 1.9 \pm 0.3 \AA$ and a correlation length of $L_{S X W} \simeq 2.9 \pm 0.2 \AA$, while the red curve is the best fit using Eq. (3) and yields a roughness of $\Delta_{C M} \simeq 0.2 \mathrm{~nm}$ and a correlation length of $L_{C M} \simeq 0.8 \mathrm{~nm}$. As shown above, STM reveals roughness values of $\Delta \simeq 0.1 \mathrm{~nm}$ and $L \simeq 1 \mathrm{~nm}$, which almost perfectly agree with those of the $\mathrm{CM}$ model. Any height dependence of $\Delta$ and $L$ is neglected in this discussion. At least for the thinnest wires we were able to show that the roughness parameters, which we discussed in the context of Fig. 3, are quite representative for most of the wires.

The trend to underestimate the correlation length in the SXW model is most likely ascribable to its assumptions and certain boundary conditions. Indeed, Eq. (2) is valid only in the limit of small correlation lengths [77], or more precisely until $k_{1} L \ll 1$ [81] (where $k_{1}$ is the largest of the Fermi wave vectors $k_{n}$ of subband $n$ ), which is not fulfilled in our case. Moreover, Fishman et al. [85] showed that the surface contribution within Eq. (2) strongly changes when $k_{1} L \gg 1$ and needs to be replaced by an $n \times n$ matrix, which they explicitly evaluated for the surface scattering term only (i.e., neglecting the contribution coming from the bulk). Although incomplete, this analysis showed that the surface scattering decreases by several orders of magnitude for high quality surfaces (i.e., $L \gg 1 \mathrm{~nm}$ ). Thus a pronounced decrease in the resistivity of as-grown $\mathrm{TbSi}_{2}$ is expected, if the growth process is stopped once a complete layer is formed. This is also evident from the semiclassical description of Chatterjee and Meyerovich, where the leading terms in Eq. (3) reveal a $1 / L$ dependence. We finally point out that the SXW description is strictly valid only in the limit of the white-noise approximation (i.e., assuming a quasiuncorrelated surface or $L \ll 1 \mathrm{~nm}$ ) [86]. For a correlated surface roughness Munoz derived a new expression (named modified SXW) based on weaker constriction $\left(\Delta^{2} k_{F} \ll L\right)$, but relying on low temperatures [86].

\section{SUMMARY AND CONCLUSION}

In this paper we reported on the growth of $\mathrm{TbSi}_{2}$ wires and comprehensively characterized these one-dimensional structures by means of a multitip STM/SEM system. Our experimental setup allowed us to analyze the transport properties of individual $\mathrm{Tb}$ silicide wires and correlate the transport findings with the atomic structure. Depending on details of the preparation, several classes of wires were found with distinct resistance values per lengths. In contrast to their widths, the heights of the wires vary by an order of magnitude being comparable or even smaller than the characteristic electron mean free path. Most importantly, we showed that the resistivity values of these wires are strongly increasing with decreasing height. Albeit our experimental parameters cannot be entirely considered by current theories, we succeeded in a quantitative description of this finding on the basis of the Chatterjee and Meyerovich model [31], showing both the importance of surface and bulk channels as well as of the atomistic roughness on these wires.

\section{ACKNOWLEDGMENTS}

Financial support by the Deutsche Forschungsgemeinschaft (DFG) through the FOR1700, projects E2, E4, and T1, is gratefully acknowledged.

Author contributions. I.M. and F.E. equally contributed to this work. I.M., F.E., and C.T. conceived and designed the experiments. The growth experiments were performed by F.E., I.M. and S.A. The DFT calculations were done by K.H., S.S. and W.G.S. The manuscript has been written by F.E., I.M., and C.T. All authors reviewed the manuscript and have given approval to the final version of the manuscript.
[1] W. Tang, S. A. Dayeh, S. T. Picraux, J. Y. Huang, and K.-N. Tu, Nano Lett. 12, 3979 (2012).

[2] S.-Y. Chen, P.-H. Yeh, W.-W. Wu, U.-S. Chen, Y.-L. Chueh, Y.-C. Yang, S. Gwo, and L.-J. Chen, ACS Nano 5. 9202 (2011).

[3] A. L. Schmitt, J. M. Higgins, J. R. Szczech, and S. Jin, J. Mater. Chem. 20, 223 (2010).

[4] C. Zeng, P. R. C. Kent, T.-H. Kim, A.-P. Li, and H. H. Weitering, Nat. Mater. 7, 539 (2008).

[5] V. Iancu, X.-G. Zhang, T.-H. Kim, L. D. Menard, P. R. C. Kent, M. E. Woodson, J. M. Ramsey, A.-P. Li, and H. H. Weitering, Nano Lett. 13, 3684 (2013).

[6] S. Qin, T.-H. Kim, Y. Zhang, W. Ouyang, H. H. Weitering, C.-K. Shih, A. P. Baddorf, R. Wu, and A.-P. Li, Nano Lett. 12, 938 (2012).
[7] Silicide Technology for Integrated Circuits, edited by L. J. Chen (IET, New York, 2004), Vol. 5.

[8] P. S. Y. Lim, D. Z. Chi, Q. Zhou, and Y.-C. Yeo, J. Appl. Phys. 113, 013712 (2013).

[9] The International Technology Roadmap for Semiconductors, Tech. Rep., ITRS, 2007, http://www.itrs2.net/itrs-reports.html.

[10] S.-L. Zhang and Z. Zhang, in Metallic Films for Electronic, Optical and Magnetic Applications, edited by K. Barmak and K. Coffey (Woodhead Publishing, New York, 2014), p. 244.

[11] I.-H. Hong, T.-M. Chen, and Y.-F. Tsai, Appl. Surf. Sci. 349, 49 (2015).

[12] G. Ottaviani, K. N. Tu, and J. W. Mayer, Phys. Rev. Lett. 44, 284 (1980).

[13] K. N. Tu, R. D. Thompson, and B. Y. Tsaur, Appl. Phys. Lett. 38, 626 (1981). 
[14] L. Chen and K. Tu, Mater. Sci. Rep. 6, 53 (1991).

[15] Y. Chen, D. A. A. Ohlberg, and R. S. Williams, J. Appl. Phys. 91, 3213 (2002).

[16] J. L. McChesney, A. Kirakosian, R. Bennewitz, J. N. Crain, J.-L. Lin, and F. J. Himpsel, Nanotechnology 13, 545 (2002).

[17] M. Franz, J. Große, R. Kohlhaas, and M. Dähne, Surf. Sci. 637-638, 149 (2015).

[18] G. Grüner, Rev. Mod. Phys. 66, 1 (1994).

[19] Y. Chen, D. a. A. Ohlberg, G. Medeiros-Ribeiro, Y. A. Chang, and R. S. Williams, Appl. Phys. A 75, 353 (2002).

[20] C. Preinesberger, G. Pruskil, S. K. Becker, M. Dähne, D. V. Vyalikh, S. L. Molodtsov, C. Laubschat, and F. Schiller, Appl. Phys. Lett. 87, 083107 (2005).

[21] H. W. Yeom, Y. K. Kim, E. Y. Lee, K.-D. Ryang, and P. G. Kang, Phys. Rev. Lett. 95, 205504 (2005).

[22] P. Bennett, Z. He, D. J. Smith, and F. Ross, Thin Solid Films 519, 8434 (2011).

[23] M. Dähne and M. Wanke, J. Phys.: Condens. Matter 25, 014012 (2013).

[24] L. Zhigang, L. Shibing, W. Congshun, L. Ming, W. Wengang, H. Yilong, and Z. Xinwei, J. Phys. D: Appl. Phys. 39, 2839 (2006).

[25] J. Thomson, Proc. Cambridge Philos. Soc. 11, 120 (1901).

[26] K. Fuchs, Math. Proc. Cambridge Philos. Soc. 34, 100 (2008).

[27] E. H. Sondheimer, Adv. Phys. 50, 499 (2010).

[28] D. K. C. MacDonald and K. Sarginson, Proc. R. Soc. London A Mat. 203, 223 (1950).

[29] R. B. Dingle, Proc. R. Soc. London A Mat. 201, 545 (1950).

[30] L. Sheng, D. Y. Xing, and Z. D. Wang, Phys. Rev. B 51, 7325 (1995).

[31] S. Chatterjee and A. E. Meyerovich, Phys. Rev. B 81, 245409 (2010).

[32] N. Inoue, Y. Tanishiro, and K. Yagi, Jpn. J. Appl. Phys. 26, L293 (1987).

[33] B. Z. Liu and J. Nogami, Nanotechnology 14, 873 (2003).

[34] B. Z. Liu and J. Nogami, J. Appl. Phys. 93, 593 (2003).

[35] G. Kresse and J. Furthmüller, Comput. Mater. Sci. 6, 15 (1996).

[36] G. Kresse and J. Furthmüller, Phys. Rev. B 54, 11169 (1996).

[37] G. Kresse and D. Joubert, Phys. Rev. B 59, 1758 (1999).

[38] J. P. Perdew, K. Burke, and M. Ernzerhof, Phys. Rev. Lett. 77, 3865 (1996).

[39] J. P. Perdew, J. A. Chevary, S. H. Vosko, K. A. Jackson, M. R. Pederson, D. J. Singh, and C. Fiolhais, Phys. Rev. B 46, 6671 (1992).

[40] H. J. Monkhorst and J. D. Pack, Phys. Rev. B 13, 5188 (1976)

[41] S. Appelfeller, S. Kuls, and M. Dähne, Surf. Sci. 641, 180 (2015).

[42] V. Iancu, P. R. C. Kent, S. Hus, H. Hu, C. G. Zeng, and H. H. Weitering, J. Phys.: Condens. Matter 25, 014011 (2013).

[43] B. Liu and J. Nogami, Surf. Sci. 540, 136 (2003).

[44] Y. Cui, J. Chung, and J. Nogami, J. Phys.: Condens. Matter 24, 045003 (2012).

[45] A. Pratt, C. Woffinden, C. Bonet, and S. P. Tear, MRS Proc. 1144, LL04 (2008).

[46] C. Preinesberger, S. Vandré, T. Kalka, and M. Dähne-Prietsch, J. Phys. D: Appl. Phys. 31, L43 (1998).
[47] M. Degawa, H. Nishimura, Y. Tanishiro, H. Minoda, and K. Yagi, Jpn. J. Appl. Phys. 38, L308 (1999).

[48] H. Minoda, J. Phys.: Condens. Matter 15, S3255 (2003).

[49] S. Appelfeller, M. Franz, M. Kubicki, P. Rei, T. Niermann, M. A. Schubert, M. Lehmann, and M. Dähne, Appl. Phys. Lett. 108, 013109 (2016).

[50] T. Gredig, E. A. Silverstein, and M. P. Byrne, J. Phys.: Conf. Ser. 417, 012069 (2013).

[51] J. Krim and J. O. Indekeu, Phys. Rev. E 48, 1576 (1993).

[52] S. Labat, C. Guichet, O. Thomas, B. Gilles, and A. Marty, Appl. Surf. Sci. 188, 182 (2002).

[53] M. McEllistrem, G. Haase, D. Chen, and R. J. Hamers, Phys. Rev. Lett. 70, 2471 (1993).

[54] C. Ohbuchi and J. Nogami, Phys. Rev. B 66, 165323 (2002).

[55] D. Lee and S. Kim, Appl. Phys. Lett. 82, 2619 (2003).

[56] V. Iancu, P. R. C. Kent, C. G. Zeng, and H. H. Weitering, Appl. Phys. Lett. 95, 123107 (2009).

[57] M. Wanke, K. Löser, G. Pruskil, D. V. Vyalikh, S. L. Molodtsov, S. Danzenbächer, C. Laubschat, and M. Dähne, Phys. Rev. B 83, 205417 (2011).

[58] I. Miccoli, F. Edler, H. Pfnür, and C. Tegenkamp, J. Phys.: Condens. Matter 27, 223201 (2015).

[59] F. H. Kaatz, W. R. Graham, J. V. d. Spiegel, W. Joss, and J. A. Chroboczek, J. Vac. Sci. Technol. A 9, 426 (1991).

[60] H. Marom, J. Mullin, and M. Eizenberg, Phys. Rev. B 74, 045411 (2006).

[61] W. Steinhögl, G. Schindler, G. Steinlesberger, M. Traving, and M. Engelhardt, J. Appl. Phys. 97, 023706 (2005).

[62] L. Moraga, C. Arenas, R. Henriquez, and B. Solis, Phys. Status Solidi B 252, 219 (2015).

[63] A. F. Mayadas and M. Shatzkes, Phys. Rev. B 1, 1382 (1970).

[64] G. Aprilesi, E. Mazzega, M. Michelini, F. Nava, G. Queirolo, and L. Meda, J. Appl. Phys. 60, 310 (1986).

[65] C. D. Lien, M. Finetti, M.-A. Nicolet, and S. S. Lau, J. Electron. Mater. 13, 95 (1984).

[66] E. Colgan, J. Gambino, and Q. Hong, Mater. Sci. Eng., R 16, 43 (1996).

[67] J. C. Hensel, R. T. Tung, J. M. Poate, and F. C. Unterwald, Appl. Phys. Lett. 44, 913 (1984).

[68] A. F. Mayadas, M. Shatzkes, and J. F. Janak, Appl. Phys. Lett. 14, 345 (1969).

[69] G. Ye, J. Nogami, and M. A. Crimp, Thin Solid Films 497, 48 (2006).

[70] G. Ye, M. A. Crimp, and J. Nogami, J. Appl. Phys. 105, 104304 (2009).

[71] W. Steinhögl, G. Schindler, G. Steinlesberger, and M. Engelhardt, Phys. Rev. B 66, 075414 (2002).

[72] Y. P. Timalsina, A. Horning, R. F. Spivey, K. M. Lewis, T.-S. Kuan, G.-C. Wang, and T.-M. Lu, Nanotechnology 26, 075704 (2015).

[73] D. Dayal and P. Wissmann, Thin Solid Films 44, 185 (1977).

[74] H. D. Liu, Y. P. Zhao, G. Ramanath, S. P. Murarka, and G. C. Wang, Thin Solid Films 384, 151 (2001).

[75] C. Durkan and M. E. Welland, Phys. Rev. B 61, 14215 (2000).

[76] M. Jałochowski and E. Bauer, Phys. Rev. B 37, 8622 (1988).

[77] N. Trivedi and N. W. Ashcroft, Phys. Rev. B 38, 12298 (1988). 
[78] J. Ziman, Electrons and Phonons: The Theory of Transport Phenomena in Solids, International Series of Monographs on Physics (OUP, Oxford, 1960).

[79] A. Kaser and E. Gerlach, Z. Phys. B: Condens. Matter 98, 207 (1995).

[80] R. C. Munoz, C. Arenas, G. Kremer, and L. Moraga, J. Phys.: Condens. Matter 15, L177 (2003).

[81] G. Fishman and D. Calecki, Phys. Rev. Lett. 62, 1302 (1989).
[82] G. Mahan, Many-Particle Physics, Physics of Solids and Liquids (Springer, New York, 2000).

[83] Z. Tešanović, M. V. Jarić, and S. Maekawa, Phys. Rev. Lett. 57, 2760 (1986).

[84] S. Chatterjee and A. E. Meyerovich, Phys. Rev. B 84, 165432 (2011).

[85] G. Fishman and D. Calecki, Phys. Rev. B 43, 11581 (1991).

[86] R. C. Munoz, J. Mol. Catal. A: Chem. 228, 163 (2005). 